\title{
Point Sets in the Unit Square and Large Areas of Convex Hulls of Subsets of Points
}

\author{
Hanno Lefmann \\ Fakultät für Informatik, TU Chemnitz, D-09107 Chemnitz, Germany \\ lefmann@informatik.tu-chemnitz.de
}

\begin{abstract}
In this paper generalizations of Heilbronn's triangle problem are considered. By using results on the independence number of linear hypergraphs, for fixed integers $k \geq 3$ and any integers $n \geq k$ a $o\left(n^{6 k-4}\right)$ time deterministic algorithm is given, which finds distributions of $n$ points in the unit square $[0,1]^{2}$ such that, simultaneously for $j=3, \ldots, k$, the areas of the convex hulls determined by any $j$ of these $n$ points are $\Omega\left((\log n)^{1 /(j-2)} / n^{(j-1) /(j-2)}\right)$.
\end{abstract}

\section{Introduction}

Distributions of $n$ points in the unit square $[0,1]^{2}$ such that the minimum area of a triangle determined by three of these $n$ points is large have been investigated by Heilbronn. Let $\Delta_{3}(n)$ denote the supremum over all distributions of $n$ points in $[0,1]^{2}$ of the minimum area of a triangle among $n$ points. Since no three of the points $1 / n \cdot\left(i \bmod n, i^{2} \bmod n\right), i=0, \ldots, n-1$, are collinear, we infer $\Delta_{3}(n)=\Omega\left(1 / n^{2}\right)$, provided $n$ is prime, as has been observed by Erdös. For a while this lower bound was believed to be also the upper bound. However, Komlós, Pintz and Szemerédi [14] proved that $\Delta_{3}(n)=\Omega\left(\log n / n^{2}\right)$, see [7] for a deterministic polynomial time algorithm achieving this lower bound. Upper bounds on $\Delta_{3}(n)$ were given by Roth [19]-[22] and Schmidt [23] and, improving these earlier results, the currently best upper bound $\Delta_{3}(n)=O\left(2^{c \sqrt{\log n}} / n^{8 / 7}\right)$ for a constant $c>0$, is due to Komlós, Pintz and Szemerédi [13]. We remark that the expected value of the minimum area of a triangle formed by three of $n$ uniformly at random and independently of each other distributed points in $[0,1]^{2}$ has been shown in [12] to be equal to $\Theta\left(1 / n^{3}\right)$.

Variants of Heilbronn's triangle problem in higher dimensions were investigated by Barequet [3,4], who considered the minimum volumes of simplices among $n$ points in the $d$-dimensional unit cube $[0,1]^{d}$, see also [15] and Brass [8]. Recently, Barequet and Shaikhet [5] considered the on-line situation, where the points have to be positioned one after the other and suddenly this process stops. For this situation they obtained for the supremum of the minimum volume of $(d+1)$-point simplices among $n$ points in $[0,1]^{d}$ the lower bound $\Omega\left(1 / n^{(d+1)} \ln (d-2)+2\right)$.

A generalization of Heilbronn's triangle problem to $k$-gons, see Schmidt [23], asks, given an integer $k \geq 3$, to maximize the minimum area of the convex hull of any $k$ distinct points in a distribution of $n$ points in $[0,1]^{2}$. In particular, let $\Delta_{k}(n)$ be the supremum over all distributions of $n$ points in $[0,1]^{2}$ 
of the minimum area of the convex hull determined by some $k$ of $n$ points. For $k=4$, Schmidt [23] proved the lower bound $\Delta_{4}(n)=\Omega\left(1 / n^{3 / 2}\right)$, and in [7] the lower bound $\Delta_{k}(n)=\Omega\left(1 / n^{(k-1) /(k-2)}\right)$ has been shown for fixed integers $k \geq 3$. Also in [7] a deterministic polynomial time algorithm was given which achieves this lower bound. This has been improved in [16] to $\Delta_{k}(n)=$ $\Omega\left((\log n)^{1 /(k-2)} / n^{(k-1) /(k-2)}\right)$ for any fixed integers $k \geq 3$.

We remark that for $k$ a function of $n$, Chazelle proved in [9] in connection with some range searching problems $\Delta_{k}(n)=\Theta(k / n)$ for $\log n \leq k \leq n$.

In [17] a deterministic polynomial time algorithm has been given, which finds for fixed integers $k \geq 2$ and any integers $n \geq k$ a distribution of $n$ points in the unit square $[0,1]^{2}$ such that, simultaneously for $j=2, \ldots, k$, the areas of the convex hulls of any $j$ among the $n$ points are $\Omega\left((\log n)^{1 /(j-2)} / n^{(j-1) /(j-2)}\right)$. Recently, in [18] these (simultaneously achievable) lower bounds on the minimum areas of the convex hull of any $j$ among $n$ points in $[0,1]^{2}$ have been improved by using (non-discrete) probabilistic arguments by a polylogarithmic factor to $\Omega\left((\log n)^{1 /(j-2)} / n^{(j-1) /(j-2)}\right)$ for $j=3, \ldots, k$. (Note that $\Delta_{2}(n)=\Theta\left(1 / n^{1 / 2}\right)$.) While this was an existence argument, here we give a deterministic polynomial time algorithm, which provides such a configuration of $n$ points in $[0,1]^{2}$.

Theorem 1. Let $k \geq 3$ be a fixed integer. For each integer $n \geq k$ one can find deterministically in time o( $\left.n^{6 k-4}\right)$ some $n$ points in the unit square $[0,1]^{2}$ such that, simultaneously for $j=3, \ldots, k$, the minimum area of the convex hull determined by some $j$ of these $n$ points is $\Omega\left((\log n)^{1 /(j-2)} / n^{(j-1) /(j-2)}\right)$.

Concerning upper bounds, we remark that for fixed $j \geq 4$ only the simple bounds $\Delta_{j}(n)=O(1 / n)$ are known, compare [23].

\section{The Independence Number of Linear Hypergraphs}

In our considerations we transform the geometric problem into a problem on hypergraphs.

Definition 1. A hypergraph is a pair $\mathcal{G}=(V, \mathcal{E})$ with vertex-set $V$ and edgeset $\mathcal{E}$, where $E \subseteq V$ for each edge $E \in \mathcal{E}$. For a hypergraph $\mathcal{G}$ the notation $\mathcal{G}=\left(V, \mathcal{E}_{2} \cup \cdots \cup \mathcal{E}_{k}\right)$ means that $\mathcal{E}_{i}$ is the set of all i-element edges in $\mathcal{G}$, $i=2, \ldots, k$. A hypergraph $\mathcal{G}=(V, \mathcal{E})$ is called $k$-uniform if $|E|=k$ for each edge $E \in \mathcal{E}$. The independence number $\alpha(\mathcal{G})$ of $\mathcal{G}=(V, \mathcal{E})$ is the largest size of a subset $I \subseteq V$ which contains no edges from $\mathcal{E}$.

For hypergraphs $\mathcal{G}$ a lower bound on the independence number $\alpha(\mathcal{G})$ is given by Turán's theorem for hypergraphs, see [24].

Theorem 2. Let $\mathcal{G}=\left(V, \mathcal{E}_{2} \cup \cdots \cup \mathcal{E}_{k}\right)$ be a hypergraph on $|V|=N$ vertices with average degree $t_{i}^{i-1}:=i \cdot\left|\mathcal{E}_{i}\right| /|V|$ for the $i$-element edges, $i=2, \ldots, k$. Let $t_{i_{0}}:=\max \left\{t_{i} \mid 2 \leq i \leq k\right\} \geq 1 / 2$.

Then, the independence nunber $\alpha(\mathcal{G})$ of $\mathcal{G}$ satisfies

$$
\alpha(\mathcal{G}) \geq N /\left(4 \cdot t_{i_{0}}\right)
$$


An independent set $I \subseteq V$ in $\mathcal{G}$ with $|I| \geq N /\left(4 \cdot t_{i_{0}}\right)$ can be found deterministically in time $O\left(|V|+\left|\mathcal{E}_{2}\right|+\cdots+\left|\mathcal{E}_{k}\right|\right)$.

For fixed positive integers $k \geq 2$ one can show by Theorem 2 and Lemmas 2 and 4 (see below), that one can find deterministically in polynomial time $n$ points in $[0,1]^{2}$ such that the areas of the convex hulls of any $j$ of these $n$ points are $\Omega\left(1 / n^{(j-1) /(j-2)}\right)$ simultaneously for $j=2, \ldots, k$, compare [17]. However, we want to obtain better lower bounds. To achieve this, we consider the independence number of hypergraphs, which do not contain cycles of small lenghts.

Definition 2. A j-cycle in a hypergraph $\mathcal{G}=(V, \mathcal{E})$ is a sequence $E_{1}, \ldots, E_{j}$ of distinct edges $E_{1}, \ldots, E_{j} \in \mathcal{E}$, such that $E_{i} \cap E_{i+1} \neq \emptyset$ for $i=1, \ldots, j-1$, and $E_{j} \cap E_{1} \neq \emptyset$, and a sequence $v_{1}, \ldots, v_{j}$ of distinct vertices with $v_{i+1} \in \mathcal{E}_{i} \cap \mathcal{E}_{i+1}$ for $i=1, \ldots, j-1$, and $v_{1} \in \mathcal{E}_{1} \cap \mathcal{E}_{j}$. An unordered pair $\left\{E, E^{\prime}\right\}$ of distinct edges $E, E^{\prime} \in \mathcal{E}$ with $\left|E \cap E^{\prime}\right| \geq 2$ is called a 2-cycle. For a hypergraph $\mathcal{G}=$ $\left(V, \mathcal{E}_{3} \cup \cdots \cup \mathcal{E}_{k}\right)$ a 2-cycle $\left\{E, E^{\prime}\right\}$ in $\mathcal{G}$ is called $(2 ;(g, i, j))$-cycle if and only if $\left|E \cap E^{\prime}\right|=g$, and $E \in \mathcal{E}_{i}$ and $E^{\prime} \in \mathcal{E}_{j}$ for $2 \leq g \leq i \leq j$ but $g<j$. A hypergraph $\mathcal{G}=(V, \mathcal{E})$ is called linear if it does not contain any 2 -cycles, and it is called uncrowded if it does not contain any 2-, 3-or 4-cycles.

For $k$-uniform uncrowded hypergraphs the next lower bound on the independence number, which has been proved by Ajtai, Komlós, Pintz, Spencer and Szemerédi [1], is better than the one in (1), see also [2] and [10], and compare [6] and [11] for a deterministic polynomial time algorithm.

Theorem 3. Let $k \geq 3$ be a fixed integer. Let $\mathcal{G}=\left(V, \mathcal{E}_{k}\right)$ be an uncrowded $k$ uniform hypergraph with $|V|=N$ vertices and average degree $t^{k-1}:=k \cdot\left|\mathcal{E}_{k}\right| / N$. Then, for some constant $C_{k}>0$ the independence number $\alpha(\mathcal{G})$ of $\mathcal{G}$ satisfies

$$
\alpha(\mathcal{G}) \geq C_{k} \cdot(N / t) \cdot(\log t)^{\frac{1}{k-1}} .
$$

Hence, for fixed integers $k \geq 3$ and uncrowded $k$-uniform hypergraphs with average degree $t^{k-1}$ the lower bound (2) improves (1) by a factor of $\Theta\left((\log t)^{1 /(k-1)}\right)$. We use the following extension of Theorem 3 - instead of an uncrowded hypergraph we require only a linear one -, see [17].

Theorem 4. Let $k \geq 3$ be a fixed integer. Let $\mathcal{G}=\left(V, \mathcal{E}_{3} \cup \cdots \cup \mathcal{E}_{k}\right)$ be a linear hypergraph with $|V|=N$ such that the average degrees $t_{i}^{i-1}:=i \cdot\left|\mathcal{E}_{i}\right| /|V|$ for the $i$-element edges satisfy $t_{i}^{i-1} \leq c_{i} \cdot S^{i-1} \cdot(\log S)^{(k-i) /(k-1)}$, where $c_{i}>0$ are constants with $c_{i}<1 / 32 \cdot\left(\begin{array}{c}k-1 \\ i-1\end{array}\right) /\left(10^{(3(k-i)) /(k-1)} \cdot k^{6}\right), i=3, \ldots, k$.

Then for some constant $C_{k}>0$, the independence number $\alpha(\mathcal{G})$ of $\mathcal{G}$ satisfies

$$
\alpha(\mathcal{G}) \geq C_{k} \cdot \frac{N}{S} \cdot(\log S)^{\frac{1}{k-1}} .
$$

An independent set of size $\Omega\left((N / S) \cdot(\log S)^{1 /(k-1)}\right)$ can be found deterministically in time $O\left(N \cdot S^{4 k-2}\right)$. 
Both Theorems 3 and 4 are best possible for a certain range of the parameters $k<T<N$ as can be seen by a random hypergraph argument.

Theorem 4 is helpful in our situation, since one has to take care only of the 2-cycles and not of 3- and 4-cycles anymore.

\section{A Deterministic Algorithm}

Here we prove Theorem 1 . To give a polynomial time algorithm, which for fixed integers $k \geq 3$ finds for any integers $n \geq k$ deterministically $n$ points in the unit square $[0,1]^{2}$ such that simultaneously for $j=3, \ldots, k$, the areas of the convex hulls of any $j$ of these $n$ points are $\Omega\left((\log n)^{1 /(j-2)} / n^{(j-1) /(j-2)}\right)$, we discretize the unit square $[0,1]^{2}$ by considering the standard $T \times T$-grid, i.e., the set $\left\{(i, j) \in \mathbb{Z}^{2} \mid 0 \leq i, j \leq T-1\right\}$, where $T=n^{1+\beta}$ for some constant $\beta>0$, which will be specified later.

For distinct grid-points $P, Q$ in the $T \times T$-grid let $P Q$ denote the line through $P$ and $Q$ and let $[P, Q]$ denote the segment between $P$ and $Q$. Let dist $(P, Q):=$ $\left(\left(p_{x}-q_{x}\right)^{2}+\left(p_{y}-q_{y}\right)^{2}\right)^{1 / 2}$ denote the Euclidean distance between the grid-points $P=\left(p_{x}, p_{y}\right)$ and $Q=\left(q_{x}, q_{y}\right)$. For grid-points $P_{1}, \ldots, P_{l}$ in the $T \times T$-grid let area $\left(P_{1}, \ldots, P_{l}\right)$ be the area of the convex hull of the points $P_{1}, \ldots, P_{l}$. A strip centered at the line $P Q$ of width $w$ is the set of all points in $\mathbb{R}^{2}$, which are at Euclidean distance at most $w / 2$ from the line $P Q$. Let $\leq_{l}$ be a total order on the $T \times T$-grid, which is defined as follows: for grid-points $P=\left(p_{x}, p_{y}\right)$ and $Q=$ $\left(q_{x}, q_{y}\right)$ in the $T \times T$-grid let $P \leq_{l} Q: \Longleftrightarrow\left(p_{x}<q_{x}\right)$ or $\left(p_{x}=q_{x}\right.$ and $\left.p_{y}<q_{y}\right)$. First notice the following simple fact.

Lemma 1. Let $P_{1}, \ldots, P_{l}$ be grid-points in the $T \times T$-grid, $l \geq 3$.

(i) Then, it is area $\left(P_{1}, \ldots, P_{l}\right) \geq$ area $\left(P_{1}, \ldots, P_{l-1}\right)$.

(ii) If area $\left(P_{1}, \ldots, P_{l}\right) \leq A$, then for any distinct grid-points $P_{i}, P_{j}$ every gridpoint $P_{k}, k=1, \ldots, l$, is contained in a strip centered at the line $P_{i} P_{j}$ of width $(4 \cdot A) / \operatorname{dist}\left(P_{i}, P_{j}\right)$.

For suitable constants $c_{j}^{*}>0, j=3, \ldots, k$, we set

$$
A_{j}:=\frac{c_{j}^{*} \cdot T^{2} \cdot(\log n)^{1 /(j-2)}}{n^{(j-1) /(j-2)}}>1 .
$$

Then, it is $0<A_{3} \leq \cdots \leq A_{k}$ for $n \geq n_{0}$. We form a hypergraph $\mathcal{G}=$ $\mathcal{G}\left(A_{3}, \ldots, A_{k}\right)=\left(V, \overline{\mathcal{E}_{3}^{0}} \cup \mathcal{E}_{3} \cup \mathcal{E}_{4} \cup \cdots \cup \overline{\mathcal{E}}_{k}\right)$, which contains two types of 3 element edges, and (one type of) $j$-element edges, $j=4, \ldots, k$. The vertex-set $V$ of $\mathcal{G}$ consists of the $T^{2}$ grid-points in the $T \times T$-grid. The edge-sets are defined as follows. For distinct grid-points $P, Q, R \in V$ in the $T \times T$-grid let $\{P, Q, R\} \in \mathcal{E}_{3}^{0}$ if and only if $P, Q, R$ are collinear. Moreover, for $j=3, \ldots, k$, and distinct gridpoints $P_{1}, \ldots, P_{j} \in V$ in the $T \times T$-grid let $\left\{P_{1}, \ldots, P_{j}\right\} \in \mathcal{E}_{j}$ if and only if area $\left(P_{1}, \ldots, P_{j}\right) \leq A_{j}$ and no three of the grid-points $P_{1}, \ldots, P_{j}$ are collinear. 
We want to find a large independent set in this hypergraph $\mathcal{G}=\left(V, \mathcal{E}_{3}^{0} \cup \mathcal{E}_{3} \cup\right.$ $\mathcal{E}_{4} \cup \cdots \cup \mathcal{E}_{k}$ ), as an independent set $I \subseteq V$ in $\mathcal{G}$ corresponds to $|I|$ many gridpoints in the $T \times T$-grid, such that the areas of the convex hulls of any $j$ distinct grid-points from these $|I|$ points are bigger than $A_{j}, j=3, \ldots, k$. To find a suitable induced subhypergraph of $\mathcal{G}$ to which Theorem 4 may be applied, in a first step we estimate the numbers $\left|\mathcal{E}_{3}^{0}\right|$ and $\left|\mathcal{E}_{j}\right|, j=3, \ldots, k$, of 3 - and $j$-element edges, respectively, and the numbers of 2-cycles in $\mathcal{G}$. Then in a certain induced subhypergraph $\mathcal{G}^{*}$ of $\mathcal{G}$ we omit one vertex from each 3 -element edge in $\mathcal{E}_{3}^{0}$ and from each 2-cycle. The resulting induced subhypergraph $\mathcal{G}^{* *}$ contains no 2-cycles anymore, hence is linear, and then we may apply Theorem 4 to $\mathcal{G}^{* *}$.

\subsection{The Numbers of Edges in $\mathcal{G}$}

The next estimate is quite crude but it suffices for our purposes.

Lemma 2. The number $\left|\mathcal{E}_{3}^{0}\right|$ of 3-element edges in the hypergraph $\mathcal{G}=\left(V, \mathcal{E}_{3}^{0} \cup\right.$ $\left.\mathcal{E}_{3} \cup \mathcal{E}_{4} \cup \cdots \cup \mathcal{E}_{k}\right)$ satisfies

$$
\left|\mathcal{E}_{3}^{0}\right| \leq T^{5} .
$$

Proof. For grid-points $P, Q, R \in V$ we have $\{P, Q, R\} \in \mathcal{E}_{3}^{0}$ if and only if $P, Q, R$ are collinear. Each line is determined by two grid-points in the $T \times T$-grid, for which there are at most $T^{2}$ choices each, and each line contains at most $T$ gridpoints, and the upper bound $T^{5}$ on the number of collinear triples follows.

To estimate $\left|\mathcal{E}_{j}\right|, j=3, \ldots, k$, we use the following result from [7].

Lemma 3. For distinct grid-points $P=\left(p_{x}, p_{y}\right)$ and $R=\left(r_{x}, r_{y}\right)$ with $P \leq_{l} R$ from the $T \times T$-grid, where $s:=r_{x}-p_{x} \geq 0$ and $h:=r_{y}-p_{y}$, it holds:

(a) There are at most $4 \cdot A$ grid-points $Q$ in the $T \times T$-grid such that

(i) $P \leq_{l} Q \leq_{l} R$, and

(ii) $P, Q, R$ are not collinear, and area $(P, Q, R) \leq A$.

(b) The number of grid-points $Q$ in the $T \times T$-grid which fulfill only (ii) from (a) is at most $(12 \cdot A \cdot T) / s$ for $s>0$, and at most $(12 \cdot A \cdot T) /|h|$ for $|h|>s$.

Lemma 4. For $j=3, \ldots, k$, the numbers $\left|\mathcal{E}_{j}\right|$ of unordered $j$-tuples $P_{1}, \ldots, P_{j}$ of distinct grid-points in the $T \times T$-grid with area $\left(P_{1}, \ldots, P_{j}\right) \leq A_{j}$, where no three of the grid-points $P_{1}, \ldots, P_{j}$ are collinear, satisfy for some constants $c_{j}>0$ :

$$
\left|\mathcal{E}_{j}\right| \leq c_{j} \cdot A_{j}^{j-2} \cdot T^{4} .
$$

Proof. Let $P_{1}, \ldots, P_{j}$ be grid-points, no three on a line, in the $T \times T$-grid with area $\left(P_{1}, \ldots, P_{j}\right) \leq A_{j}$. We may assume that $P_{1} \leq_{l} \cdots \leq_{l} P_{j}$. For $P_{1}=$ $\left(p_{1, x}, p_{1, y}\right)$ and $P_{k}=\left(p_{j, x}, p_{j, y}\right)$ let $s:=p_{j, x}-p_{1, x} \geq 0$ and $h:=p_{j, y}-p_{1, y}$. Then $s>0$, as otherwise $P_{1}, \ldots, P_{j}$ are collinear.

There are $T^{2}$ choices for the grid-point $P_{1}$. Given $P_{1}$, any grid-point $P_{j}$ with $P_{1} \leq{ }_{l} P_{j}$ is determined by a pair $(s, h) \neq(0,0)$ of integers with $1 \leq s \leq T$ and 
$-T \leq h \leq T$. By Lemma 1 (i) we have area $\left(P_{1}, P_{i}, P_{j}\right) \leq A_{j}$ for $i=2, \ldots, j-1$. Given the grid-points $P_{1}$ and $P_{j}$, since $P_{1} \leq_{l} P_{i} \leq_{l} P_{j}$ for $i=2, \ldots, j-1$, by Lemma 3 (a) there are at most $4 \cdot A_{j}$ choices for each grid-point $P_{i}$, hence for a constant $c_{j}>0$ :

$$
\left|\mathcal{E}_{j}\right| \leq T^{2} \cdot \sum_{s=1}^{T} \sum_{h=-T}^{T}\left(4 \cdot A_{j}\right)^{j-2} \leq c_{j} \cdot A_{j}^{j-2} \cdot T^{4} .
$$

By (6) the average degrees $t_{j}^{j-1}$ for the $j$-element edges $E \in \mathcal{E}_{j}, j=3, \ldots, k$, of $\mathcal{G}$ satisfy

$$
t_{j}^{j-1}=j \cdot\left|\mathcal{E}_{j}\right| /|V| \leq j \cdot c_{j} \cdot A_{j}^{j-2} \cdot T^{2}=:\left(t_{j}(0)\right)^{j-1} .
$$

\subsection{The Numbers of 2-Cycles in $\mathcal{G}$}

Let $s_{2 ;(g, i, j)}(\mathcal{G})$ denote the number of $(2 ;(g, i, j))$-cycles, $2 \leq g \leq i \leq j \leq k$ with $g<j$ in the hypergraph $\mathcal{G}$, i.e., the number of unordered pairs $\left\{E, E^{\prime}\right\}$ of edges with $E \in \mathcal{E}_{i}$ and $E^{\prime} \in \mathcal{E}_{j}$ and $\left|E \cap E^{\prime}\right|=g$.

Lemma 5. For $2 \leq g \leq i \leq j \leq k$ with $g<j$, there exist constants $c_{2 ;(g, i, j)}>0$ such that the numbers $s_{2 ;(g, i, j)}(\mathcal{G})$ of $(2 ;(g, i, j))$-cycles in the hypergraph $\mathcal{G}=$ $\left(V, \mathcal{E}_{3}^{0} \cup \mathcal{E}_{3} \cup \mathcal{E}_{4} \cup \cdots \cup \mathcal{E}_{k}\right)$ fulfill

$$
s_{2 ;(g, i, j)}(\mathcal{G}) \leq c_{2 ;(g, i, j)} \cdot A_{i}^{i-2} \cdot A_{j}^{j-g} \cdot T^{4} \cdot(\log T)^{3} .
$$

Proof. Let the grid-points, which correspond to the vertices of an $i$-element edge $E \in \mathcal{E}_{i}$ and a $j$-element edge $E^{\prime} \in \mathcal{E}_{j}$ and also yield a $(2 ;(g, i, j))$-cycle in $\mathcal{G}$, $2 \leq g \leq i \leq j \leq k$ with $g<j$, be $P_{1}, \ldots, P_{i}$ and $P_{1}, \ldots, P_{g}, Q_{g+1}, \ldots, Q_{j}$, where after renumbering $P_{1} \leq_{l} \cdots \leq_{l} P_{g}$ and no three of the grid-points $P_{1}, \ldots, P_{i}$ and of $P_{1}, \ldots, P_{g}, Q_{g+1}, \ldots, Q_{j}$ are collinear, thus area $\left(P_{1}, \ldots, P_{i}\right) \leq A_{i}$ and area $\left(P_{1}, \ldots, P_{g}, Q_{g+1}, \ldots, Q_{j}\right) \leq A_{j}$.

There are $T^{2}$ choices for the grid-point $P_{1}$. Given the grid-point $P_{1}=\left(p_{1, x}, p_{1, y}\right)$, any pair $(s, h) \neq(0,0)$ of integers determines at most one grid-point $P_{g}=$ $\left(p_{1, x}+s, p_{1, y}+h\right)$ in the $T \times T$-grid. By symmetry we may assume that $s>0$ and $0 \leq h \leq s \leq T$, which is taken into account by an additional constant factor $c^{\prime}>1$. Given the grid-points $P_{1}$ and $P_{g}$, since area $\left(P_{1}, P_{f}, P_{g}\right) \leq A_{i}$ for $f=2, \ldots, g-1$ by Lemma 1 , and $P_{1} \leq_{l} P_{f} \leq_{l} P_{g}$, by Lemma 3 (a) there are at most $4 \cdot A_{i}$ choices for each grid-point $P_{f}$ in the $T \times T$-grid, hence the number of choices for the grid-points $P_{1}, \ldots, P_{g-1}$ is at most

$$
T^{2} \cdot\left(4 \cdot A_{i}\right)^{g-2} .
$$

For the convex hulls of the grid-points $P_{1}, \ldots, P_{i}$ and $P_{1}, \ldots, P_{g}, Q_{g+1}, \ldots, Q_{j}$ let their (w.r.t $\leq_{l}$ ) extremal points be $P^{\prime}, P^{\prime \prime} \in\left\{P_{1}, \ldots, P_{i}\right\}$ and $Q^{\prime}, Q^{\prime \prime} \in$ $\left\{P_{1}, \ldots, P_{g}, Q_{g+1}, \ldots, Q_{j}\right\}$, respectively, i.e., for $P^{\prime} \leq_{l} P^{\prime \prime}$ and $Q^{\prime} \leq_{l} Q^{\prime \prime}$ we have $P^{\prime} \leq_{l} P_{1}, \ldots, P_{i} \leq_{l} P^{\prime \prime}$ and $Q^{\prime} \leq_{l} P_{1}, \ldots, P_{g}, Q_{g+1}, \ldots, Q_{j} \leq_{l} Q^{\prime \prime}$.

Given the grid-points $P_{1} \leq_{l} \cdots \leq_{l} P_{g}$, there are three possibilities for the convex hulls of the grid-points $P_{1}, \ldots, P_{i}$ and $P_{1}, \ldots, P_{j}, Q_{j+1}, \ldots, Q_{k}$ each: 
(i) $P_{1}$ and $P_{g}$ are extremal, or

(ii) exactly one grid-point, $P_{1}$ or $P_{g}$, is extremal, or

(iii) neither $P_{1}$ nor $P_{g}$ is extremal.

We restrict our calculations to the convex hull of $P_{1}, \ldots, P_{i}$ as the considerations for the convex hull of $P_{1}, \ldots, P_{g}, Q_{g+1}, \ldots, Q_{j}$ are essentially the same.

In case (i) the grid-points $P_{1}$ and $P_{g}$ are extremal for the convex hull of $P_{1}, \ldots, P_{i}$, hence $P_{1} \leq_{l} P_{g+1}, \ldots, P_{i} \leq_{l} P_{g}$. By Lemma 3 (a), since area $\left(P_{1}, P_{l}, P_{g}\right) \leq A_{i}$, $l=g+1, \ldots, i$, and no three of the grid-points $P_{1}, \ldots, P_{i}$ are collinear, there are at most $4 \cdot A_{i}$ choices for each grid-point $P_{l}$, hence the number of choices for the grid-points $P_{g+1}, \ldots, P_{i}$ is at most

$$
\text { case (i): } \quad\left(4 \cdot A_{i}\right)^{i-g} \text {. }
$$

In case (ii) exactly one of the grid-points $P_{1}$ or $P_{g}$ is extremal for the convex hull of $P_{1}, \ldots, P_{i}$. By Lemma 3 (b) there are at most $\left(12 \cdot A_{i} \cdot T\right) / s$ choices for the second extremal grid-point $P^{\prime}$ or $P^{\prime \prime}$. Having fixed this second extremal gridpoint, for each grid-point $P_{g+1}, \ldots, P_{i} \neq P^{\prime}, P^{\prime \prime}$ there are by Lemma 3 (a) at most $4 \cdot A_{i}$ choices, hence the number of choices for the grid-points $P_{g+1}, \ldots, P_{i}$ is at most

$$
\text { case (ii): } \quad\left(\left(4 \cdot A_{i}\right)^{i-g-1} \cdot 12 \cdot A_{i} \cdot T\right) / s=\left(\left(4 \cdot A_{i}\right)^{i-g} \cdot 3 \cdot T\right) / s \text {. }
$$

In case (iii) none of the grid-points $P_{1}, P_{g}$ is extremal for the convex hull of $P_{1}, \ldots, P_{i}$. By Lemma 1 (ii) all grid-points $P_{g+1}, \ldots, P_{i}$ are contained in a strip $S_{i}$, which is centered at the line $P_{1} P_{g}$, of width $\left(4 \cdot A_{i}\right) / \sqrt{h^{2}+s^{2}}$. Consider the parallelogram $\mathcal{P}_{0}=\left\{\left(p_{x}, p_{y}\right) \in S_{i} \mid p_{1, x} \leq p_{x} \leq p_{g, x}\right\}$ within the strip $S_{i}$, where $P_{1}=\left(p_{1, x}, p_{1, y}\right)$ and $P_{g}=\left(p_{g, x}, p_{g, y}\right)$ and $s=p_{g, x}-p_{1, x}$.

We divide the strip $S_{i}$ within the $T \times T$-grid into pairwise congruent parallelograms $\mathcal{P}_{0}, \mathcal{P}_{i}^{+}, \mathcal{P}_{i}^{-}, i=1, \ldots, l \leq\lfloor T / s\rfloor+2$, each of side-lengths $\left(4 \cdot A_{i}\right) / s$ and $\sqrt{h^{2}+s^{2}}$ and of area $4 \cdot A_{i}$, where for $i \geq 1$ all parallelograms $\mathcal{P}_{i}^{-}$are on the left of the parallelogram $\mathcal{P}_{0}$, and all parallelograms $\mathcal{P}_{i}^{+}$are on the right of $\mathcal{P}_{0}$, in particular $\mathcal{P}_{i}^{+}:=\left\{\left(p_{x}, p_{y}\right) \in S_{i} \mid p_{g, x}+(i-1) \cdot s \leq p_{x} \leq p_{g, x}+i \cdot s\right\}$ and $\mathcal{P}_{i}^{-}:=\left\{\left(p_{x}, p_{y}\right) \in S_{i} \mid p_{1, x}-i \cdot s \leq p_{x} \leq p_{1, x}-(i-1) \cdot s\right\}$. By Lemma 3 (a) each parallelogram $\mathcal{P}_{i}^{+}$or $\mathcal{P}_{i}^{-}$contains at most $4 \cdot A_{i}$ grid-points $P$, where $P_{1}, P_{j}, P$ are not collinear. Each extremal grid-point, $P^{\prime}$ or $P^{\prime \prime}$, is contained in some parallelogram $\mathcal{P}_{i}^{+}$or $\mathcal{P}_{i}^{-}$for some $i \geq 1$, since by our assumption neither $P_{1} \in \mathcal{P}_{0}$ nor $P_{g} \in \mathcal{P}_{0}$ are extremal. Each grid-point $P=\left(p_{x}, p_{y}\right) \in \mathcal{P}_{i}^{+} \cup \mathcal{P}_{i}^{-}, i \geq 1$, satisfies $\left|p_{x}-p_{1, x}\right| \geq i \cdot s$ or $\left|p_{x}-p_{j, x}\right| \geq i \cdot s$. Thus, if $P^{\prime} \in \mathcal{P}_{i}^{+} \cup \mathcal{P}_{i}^{-}$or $P^{\prime \prime} \in \mathcal{P}_{i}^{+} \cup \mathcal{P}_{i}^{-}$, by Lemma $3(\mathrm{~b})$ there are at most $\left(12 \cdot A_{i} \cdot T\right) /(i \cdot s)$ choices for the second extremal grid-point. Having chosen both extremal grid-points $P^{\prime}$ and $P^{\prime \prime}$ in at most $\left(4 \cdot A_{i}\right) \cdot\left(\left(12 \cdot A_{i} \cdot T\right) /(i \cdot s)\right)=\left(48 \cdot A_{i}^{2} \cdot T\right) /(i \cdot s)$ ways, for the grid-points $P_{g+1}, \ldots, P_{i} \neq P^{\prime}, P^{\prime \prime}$ there are by Lemma 3 (a) at most $\left(4 \cdot A_{i}\right)^{i-g-2}$ choices. Hence, in case (iii) the number of choices for the grid-points $P_{g+1}, \ldots, P_{i}$ is at 
most

$$
\text { case (iii): } \begin{aligned}
& \left(4 \cdot A_{i}\right)^{i-g-2} \cdot \sum_{i=1}^{\lfloor T / s\rfloor+2} \frac{48 \cdot A_{i}^{2} \cdot T}{i \cdot s}= \\
= & \left(4 \cdot A_{i}\right)^{i-g} \cdot \frac{3 \cdot T}{s} \cdot \sum_{i=1}^{\lfloor T / s\rfloor+2} \frac{1}{i} \leq\left(4 \cdot A_{i}\right)^{i-g} \cdot \frac{5 \cdot T \cdot \log T}{s} .
\end{aligned}
$$

By (10)-(12) and using $T \geq s$, in cases (i)-(iii) altogether the number of choices for the grid-points $P_{g+1}, \ldots, P_{i}$ is at most

$$
\left(4 \cdot A_{i}\right)^{i-g} \cdot\left(1+\frac{3 \cdot T}{s}+\frac{5 \cdot T \cdot \log T}{s}\right) \leq\left(4 \cdot A_{i}\right)^{i-g} \cdot \frac{9 \cdot T \cdot \log T}{s} .
$$

Similar to (13), for the number of choices of the grid-points $Q_{g+1}, \ldots, Q_{j}$ the following upper bound holds:

$$
\left(\left(4 \cdot A_{j}\right)^{j-g} \cdot 9 \cdot T \cdot \log T\right) / s .
$$

Hence with (9), (13) and (14) for $2 \leq g \leq i \leq j \leq k$ and $g<j$ we obtain for constants $c^{\prime}, c_{2 ;(g, i, j)}>0$ :

$$
\begin{aligned}
& \quad s_{2 ;(g, i, j)}(\mathcal{G}) \leq c^{\prime} \cdot T^{2} \cdot\left(4 \cdot A_{i}\right)^{g-2} \cdot \sum_{s=1}^{T} \sum_{h=0}^{s}\left(\frac{\left(4 \cdot A_{i}\right)^{i-g} \cdot 9 \cdot T \cdot \log T}{s}\right) . \\
& \quad\left(\frac{\left(4 \cdot A_{j}\right)^{j-g} \cdot 9 \cdot T \cdot \log T}{s}\right) \leq \\
& <81 \cdot c^{\prime} \cdot 4^{i+j-g-2} \cdot A_{i}^{i-2} \cdot A_{j}^{j-g} \cdot T^{4} \cdot(\log T)^{2} \cdot \sum_{s=1}^{T} \sum_{h=0}^{s} \frac{1}{s^{2}} \\
& \leq c_{2 ;(g, i, j)} \cdot A_{i}^{i-2} \cdot A_{j}^{j-g} \cdot T^{4} \cdot(\log T)^{3} .
\end{aligned}
$$

\subsection{Choosing a Subhypergraph in $\mathcal{G}$}

With probability $p:=T^{\varepsilon} / t_{k}(0) \leq 1$, hence $p=\Theta\left(T^{\varepsilon} /\left(A_{k}^{(k-2) /(k-1)} \cdot T^{2 /(k-1)}\right)\right.$ by (7), where $\varepsilon>0$ is a small constant, we pick uniformly at random and independently of each other vertices from $V$. Let $V^{*} \subseteq V$ be the random set of the picked vertices and let $\mathcal{G}^{*}=\left(V^{*}, \mathcal{E}_{3}^{0 *} \cup \mathcal{E}_{3}^{*} \cup \mathcal{E}_{4}^{*} \cup \cdots \cup \mathcal{E}_{k}^{*}\right)$ with $\mathcal{E}_{3}^{0 *}:=\mathcal{E}_{3}^{0} \cap\left[V^{*}\right]^{3}$ and $\mathcal{E}_{j}^{*}:=\mathcal{E}_{j} \cap\left[V^{*}\right]^{j}, j=3, \ldots, k$, be the on $V^{*}$ induced random subhypergraph of $\mathcal{G}$. Let $E\left[\left|V^{*}\right|\right], E\left[\left|\mathcal{E}_{3}^{0 *}\right|\right], E\left[\left|\mathcal{E}_{j}^{*}\right|\right], j=3, \ldots, k$, and $E\left[s_{2 ;(g, i, j)}\left(\mathcal{G}^{*}\right)\right], 2 \leq g \leq i \leq$ $j \leq k$ but $g<j$, be the expected numbers of vertices, collinear triples of gridpoints, $j$-element edges and $(2 ;(g, i, j))$-cycles, respectively, in $\mathcal{G}^{*}=\left(V^{*}, \mathcal{E}_{3}^{0 *} \cup\right.$ 
$\left.\mathcal{E}_{3}^{*} \cup \mathcal{E}_{4}^{*} \cup \cdots \cup \mathcal{E}_{k}^{*}\right)$. By (5), (6), and (8) we infer for constants $c_{1}^{\prime}, c_{3}^{0^{\prime}} c_{j}^{\prime}, c_{2 ;(g, i, j)}^{\prime}>0$ :

$$
\begin{aligned}
E\left[\left|V^{*}\right|\right] & =p \cdot T^{2} \geq\left(c_{1}^{\prime} \cdot T^{\frac{2 k-4}{k-1}+\varepsilon}\right) / A_{k}^{\frac{k-2}{k-1}} \\
E\left[\left|\mathcal{E}_{3}^{0 *}\right|\right] & =p^{3} \cdot\left|\mathcal{E}_{3}^{0}\right| \leq\left(c_{3}^{0^{\prime}} \cdot T^{\frac{5 k-11}{k-1}+3 \varepsilon}\right) / A_{k}^{\frac{3 k-6}{k-1}} \\
E\left[\left|\mathcal{E}_{j}^{*}\right|\right] & =p^{j} \cdot\left|\mathcal{E}_{j}\right| \leq\left(c_{j}^{\prime} \cdot T^{\frac{4 k-2 j-4}{k-1}+j \varepsilon} \cdot A_{j}^{j-2}\right) / A_{k}^{\frac{j(k-2)}{k-1}} \\
E\left[s_{2 ;(g, i, j)}\left(\mathcal{G}^{*}\right)\right] & =p^{i+j-g} \cdot s_{2 ;(g, i, j)}(\mathcal{G}) \leq \\
& \leq \frac{c_{2 ;(g, i, j)}^{\prime} \cdot T^{\frac{4 k-4-2(i+j-g)}{k-1}+\varepsilon(i+j-g)} \cdot(\log T)^{3} \cdot A_{i}^{i-2} \cdot A_{j}^{j-g}}{A_{k}^{\frac{(k-2)(i+j-g)}{k-1}}}
\end{aligned}
$$

By (15)-(18) and by Chernoff's and Markov's inequality we obtain a subhypergraph $\mathcal{G}^{*}=\left(V^{*}, \mathcal{E}_{3}^{0 *} \cup \mathcal{E}_{3}^{*} \cup \mathcal{E}_{4}^{*} \cup \cdots \cup \mathcal{E}_{k}^{*}\right)$ of $\mathcal{G}$ such that

$$
\begin{aligned}
\left|V^{*}\right| & \geq\left(\left(c_{1}^{\prime} / 2\right) \cdot T^{\frac{2 k-4}{k-1}+\varepsilon}\right) / A_{k}^{\frac{k-2}{k-1}} \\
\left|\mathcal{E}_{3}^{0 *}\right| & \leq\left(k^{3} \cdot c_{3}^{0^{\prime}} \cdot T^{\frac{5 k-11}{k-1}+3 \varepsilon}\right) / A_{k}^{\frac{3 k-6}{k-1}} \\
\left|\mathcal{E}_{j}^{*}\right| & \leq\left(k^{3} \cdot c_{j}^{\prime} \cdot T^{\frac{4 k-2 j-4}{k-1}+j \varepsilon} \cdot A_{j}^{j-2}\right) / A_{k}^{\frac{j(k-2)}{k-1}} \\
s_{2 ;(g, i, j)}\left(\mathcal{G}^{*}\right) & \leq \frac{k^{3} \cdot c_{2 ;(g, i, j)}^{\prime} \cdot T^{\frac{4 k-4-2(i+j-g)}{k-1}+\varepsilon(i+j-g)} \cdot(\log T)^{3} \cdot A_{i}^{i-2} \cdot A_{j}^{j-g}}{A_{k}^{\frac{(k-2)(i+j-g)}{k-1}}}(22)
\end{aligned}
$$

This probabilistic argument can be turned into a deterministic polynomial time algorithm by using the method of conditional probabilities. For $2 \leq g \leq i \leq j \leq$ $k$ but $g<j$, let $\mathcal{C}_{2 ;(g, i, j)}$ be the set of all $(i+j-g)$-element subsets $E \cup E^{\prime}$ of $V$ such that $E \in \mathcal{E}_{i}$ and $E^{\prime} \in \mathcal{E}_{j}$ and $\left|E \cap E^{\prime}\right|=g$. Let the grid-points in the $T \times T$-grid be $P_{1}, \ldots, P_{T^{2}}$. To each grid-point $P_{i}$ associate a variable $p_{i} \in[0,1]$, $i=1, \ldots, T^{2}$, and let $F\left(p_{1}, \ldots, p_{T^{2}}\right)$ be a function defined by

$$
\begin{aligned}
& F\left(p_{1}, \ldots, p_{T^{2}}\right):=2^{p \cdot T^{2} / 2} \cdot \prod_{i=1}^{T^{2}}\left(1-\frac{p_{i}}{2}\right)+ \\
& +\frac{\sum_{\{i, j, k\} \in \mathcal{E}_{3}^{0}} p_{i} \cdot p_{j} \cdot p_{k}}{\left(k^{3} \cdot c_{3}^{\prime} \cdot T^{\frac{5 k-11}{k-1}+3 \varepsilon}\right) / A^{\frac{3 k-6}{k-1}}}+\sum_{j=3}^{k} \frac{\sum_{\left\{i_{1}, \ldots, i_{j}\right\} \in \mathcal{E}_{j}} \prod_{l=1}^{j} p_{i_{l}}}{\left(k^{3} \cdot c_{j}^{\prime} \cdot T^{\frac{4 k-2 j-4}{k-1}+j \varepsilon} \cdot A_{j}^{j-2}\right) / A^{\frac{j(k-2)}{k-1}}}+ \\
& +\sum_{2 \leq g \leq i \leq j \leq k ; g<j} \frac{A_{k}^{\frac{(k-2)(i+j-g)}{k-1}} \cdot \sum_{\left\{i_{1}, \ldots, i_{i+j-g}\right\} \in \mathcal{C}_{j}} \prod_{l=1}^{i+j-g} p_{i_{l}}}{k^{3} \cdot c_{2 ;(g, i, j)}^{\prime} \cdot T^{\frac{4 k-4-2(i+j-g)}{k-1}+(i+j-g) \varepsilon} \cdot(\log T)^{3} \cdot A_{i}^{i-2} \cdot A_{j}^{j-g}}
\end{aligned}
$$

With the initialisation $p_{1}:=\cdots:=p_{T^{2}}:=p=T^{\varepsilon} / t_{0}$, we infer by (15)-(18) that $F(p, \ldots, p)<(2 / e)^{p T^{2} / 2}+1 / 3$, hence $F(p, \ldots, p)<1$ for $p \cdot T^{2} \geq 10$. By using the linearity of $F\left(p_{1}, \ldots, p_{T^{2}}\right)$ in each $p_{i}$, we minimize $F\left(p_{1}, \ldots, p_{T^{2}}\right)$ by choosing step by step $p_{i}:=0$ or $p_{i}:=1, i=1, \ldots, T^{2}$, and finally we achieve 
$F\left(p_{1}, \ldots, p_{T^{2}}\right)<1$. The set $V^{*}=\left\{P_{i} \in V \mid p_{i}=1\right\}$ yields an induced subhypergraph $\mathcal{G}^{*}=\left(V^{*}, \mathcal{E}_{3}^{0 *} \cup \mathcal{E}_{3}^{*} \cup \cdots \cup \mathcal{E}_{k}^{*}\right)$ of $\mathcal{G}$ with $\mathcal{E}_{i}^{*}:=\mathcal{E}_{i} \cap\left[V^{*}\right]^{i}$ for $i=3, \ldots, k$, and $\mathcal{E}_{3}^{0 *}:=\mathcal{E}_{3}^{0} \cap\left[V^{*}\right]^{3}$ which satisfies (19)-(22), as otherwise $F\left(p_{1}, \ldots, p_{T^{2}}\right)>1$ gives a contradiction. By (4)-(6) and (8) and using $T=n^{1+\beta}$ for fixed $\beta>0$, the running time of this derandomization is given by

$$
\begin{aligned}
& O\left(|V|+\left|\mathcal{E}_{3}^{0}\right|+\sum_{j=3}^{k}\left|\mathcal{E}_{j}\right|+\sum_{2 \leq g \leq i \leq j \leq k ; g<j}\left|\mathcal{C}_{2 ;(g, i, j}\right|\right)=O\left(\left|\mathcal{C}_{2 ;(2, k, k)}\right|\right)= \\
= & O\left(A_{k}^{2 k-4} \cdot T^{4} \cdot(\log T)^{3}\right)=O\left(\left(T^{4 k-4} \cdot(\log n)^{5}\right) / n^{2 k-2}\right) .
\end{aligned}
$$

Lemma 6. For each fixed $0<\varepsilon<(\beta-1) /(2 \cdot(1+\beta))$ and $\beta>1$ it is

$$
\left|\mathcal{E}_{3}^{0 *}\right|=o\left(\left|V^{*}\right|\right) .
$$

Proof. By (19), (20) and using $T=n^{1+\beta}$ with constants $\varepsilon>0$ and $\beta>1$ we have

$$
\begin{aligned}
& \left|\mathcal{E}_{3}^{0 *}\right|=o\left(\left|V^{*}\right|\right) \\
\Longleftarrow & T^{\frac{5 k-11}{k-1}+3 \varepsilon} \cdot \log T / A_{k}^{\frac{3 k-6}{k-1}}=o\left(T^{\frac{2 k-4}{k-1}+\varepsilon} / A_{k}^{\frac{k-2}{k-1}}\right) \\
\Longleftrightarrow & n^{2-(1+\beta)(1-2 \varepsilon)} \cdot(\log n)^{1-\frac{2}{k-1}}=o(1) \\
\Longleftrightarrow & (1+\beta) \cdot(1-2 \cdot \varepsilon)>2,
\end{aligned}
$$

which holds for $\varepsilon<(\beta-1) /(2 \cdot(1+\beta))$.

Lemma 7. For $2 \leq g \leq i \leq j \leq k$ but $g<j$ and each fixed $\varepsilon$ with $0<\varepsilon<$ $\frac{j-g}{(i+j-g-1)(j-2)(1+\beta)}$ it is

$$
s_{2 ;(g, i, j)}\left(\mathcal{G}^{*}\right)=o\left(\left|V^{*}\right|\right) .
$$

Proof. For $2 \leq g \leq i \leq j \leq k$ but $g<j$ by (4), (19), (22) and using $T=n^{1+\beta}$ with fixed $\beta, \varepsilon>0$ we infer

$$
\begin{aligned}
& s_{2 ;(g, i, j)}\left(\mathcal{G}^{*}\right)=o\left(\left|V^{*}\right|\right) \\
\Longleftarrow & \frac{T^{\frac{4 k-4-2(i+j-g)}{k-1}+(i+j-g) \varepsilon} \cdot(\log T)^{3} \cdot A_{i}^{i-2} \cdot A_{j}^{j-g}}{A_{k}^{\frac{(k-2)(i+j-g)}{k-1}}}=o\left(\frac{T^{\frac{2 k-4}{k-1}+\varepsilon}}{A_{k}^{\frac{k-2}{k-1}}}\right) \\
\Longleftrightarrow & n^{\varepsilon(1+\beta)(i+j-g-1)-\frac{j-g}{j-2}} \cdot(\log n)^{4+\frac{j-g}{j-2}-\frac{i+j-g-1}{k-1}}=o(1) \\
\Longleftrightarrow & \varepsilon<\frac{j-g}{(j-2)(i+j-g-1)(1+\beta)} .
\end{aligned}
$$

By setting $\varepsilon:=1 /\left(2 \cdot k^{2} \cdot(1+\beta)\right)$ and $\beta>1+1 / k^{2}$ all assumptions in Lemmas 6 and 7 and also $p=T^{\varepsilon} / t_{k}(0) \leq 1$ are fulfilled. We delete one vertex from each edge $E \in \mathcal{E}_{3}^{0 *}$, and from each 2-cycle in $\mathcal{G}^{*}$. Let $V^{* *} \subseteq V^{*}$ be the set of remaining vertices. By Lemmas 6 and 7 the induced subhypergraph $\mathcal{G}^{* *}=$ 
$\left(V^{* *}, \mathcal{E}_{3}^{* *} \cup \cdots \cup \mathcal{E}_{k}^{* *}\right)$ with $\mathcal{E}_{j}^{* *}:=\mathcal{E}_{j}^{*} \cap\left[V^{* *}\right]^{j}, j=3, \ldots, k$, where $\left|V^{* *}\right|=$ $(1-o(1)) \cdot\left|V^{*}\right| \geq\left|V^{*}\right| / 2$, contains no edges from $\mathcal{E}_{3}^{0}$ and no 2-cycles anymore, i.e., $\mathcal{G}^{* *}$ is a linear hypergraph. Since $\left|\mathcal{E}_{j}^{* *}\right| \leq\left|\mathcal{E}_{j}^{*}\right|$ with (19) and (21), the average degrees $t_{j}^{j-1}(1)$ for the $j$-element edges of $\mathcal{G}^{* *}, j=3, \ldots, k$, fulfill by (4):

$$
\begin{aligned}
t_{j}^{j-1}(1) & =\frac{j \cdot\left|\mathcal{E}_{j}^{* *}\right|}{\left|V^{* *}\right|} \leq \frac{\left(j \cdot k^{3} \cdot c_{j}^{\prime} \cdot T^{\frac{4 k-2 j-4}{k-1}+j \varepsilon} \cdot A_{j}^{j-2}\right) / A_{k}^{\frac{j(k-2)}{k-1}}}{\left(\left(c_{1}^{\prime} / 4\right) \cdot T^{\frac{2 k-4}{k-1}+\varepsilon}\right) / A_{k}^{\frac{k-2}{k-1}}} \leq \\
& \leq \frac{4 \cdot k^{4} \cdot c_{j}^{\prime} \cdot\left(c_{j}^{*}\right)^{j-2}}{c_{1}^{\prime} \cdot\left(c_{k}^{*}\right)^{\frac{(j-1)(k-2)}{k-1}}} \cdot T^{(j-1) \varepsilon} \cdot(\log n)^{\frac{k-j}{k-1}} .
\end{aligned}
$$

As observed above, this subhypergraph $\mathcal{G}^{* *}$ is linear. By choosing $S:=c \cdot T^{\varepsilon}$ for a large enough constant $c>0$, with $T=n^{1+\beta}$ with $T=n^{1+\beta}$ by (26) the assumptions in Theorem 4 are fulfilled, and we apply it, and, using (4) we find in time

$$
O\left(\left(T^{\frac{2 k-4}{k-1}+\varepsilon} / A_{k}^{\frac{k-2}{k-1}}\right) \cdot S^{4 k-2}\right)=O\left(n \cdot T^{(4 k-1) \varepsilon}\right)=o\left(T^{2}\right)
$$

an independent set $I$ of size

$$
\begin{aligned}
|I| & =\Omega\left(\left(\left|V^{* *}\right| / S\right) \cdot(\log S)^{\frac{1}{k-1}}\right)=\Omega\left(\left(T^{\frac{2 k-4}{k-1}+\varepsilon} /\left(A_{k}^{\frac{k-2}{k-1}} \cdot T^{\varepsilon}\right)\right) \cdot\left(\log T^{\varepsilon}\right)^{\frac{1}{k-1}}\right)= \\
& =\Omega\left(\left(n /(\log n)^{\frac{1}{k-1}}\right) \cdot(\log T)^{\frac{1}{k-1}}\right)=\Omega(n),
\end{aligned}
$$

since $T=n^{1+\beta}$ and $\beta, \varepsilon>0$ are constants. By choosing the constants $c_{j}^{*}>0, j=$ $3, \ldots, k$, in (4) sufficiently small, we obtain an independent set of size $n$, which yields, after rescaling the areas $A_{j}$ by the factor $T^{2}$, a desired set of $n$ points in $[0,1]^{2}$ such that, simultaneously for $j=3, \ldots, k$, the areas of the convex hulls of every $j$ distinct of these $n$ points are $\Omega\left((\log n)^{1 /(j-2)} / n^{(j-1) /(j-2)}\right)$. Adding the times in (23) and (27) we get the time bound $O\left(T^{4 k-4} \cdot(\log n)^{5} / n^{2 k-2}+T^{2}\right)=$ $\left(n^{(2 k-2)(1+2 \beta)+1)}\right)=o\left(n^{6 k-4}\right)$ for $\beta>1+1 / k^{2}$ small enough.

We remark that the bound $o\left(n^{6 k-4}\right)$ on the running time might be improved a little, for example by using a better estimate on the number of collinear triples of grid-points in the $T \times T$-grid or by a random preselection of grid-points. However, we cannot do better than $O\left(n^{c k}\right)$ for some constant $c>0$.

\section{References}

1. M. Ajtai, J. Komlós, J. Pintz, J. Spencer and E. Szemerédi, Extremal Uncrowded Hypergraphs, Journal of Combinatorial Theory Ser. A, 32, 1982, 321-335.

2. N. Alon, H. Lefmann and V. Rödl, On an Anti-Ramsey Type Result, Colloquia Mathematica Societatis János Bolyai, 60. Sets, Graphs and Numbers, 1991, 9-22.

3. G. Barequet, A Lower Bound for Heilbronn's Triangle Problem in d Dimensions, SIAM Journal on Discrete Mathematics 14, 2001, 230-236.

4. G. Barequet, The On-Line Heilbronn's Triangle Problem, Discrete Mathematics 283, 2004, 7-14. 
5. G. Barequet and A. Shaikhet, The On-Line Heilbronn's Triangle Problem in d Dimensions, Proceedings '12th Ann. Int. Computing and Combinatorics Conference COCOON'2006', LNCS 4112, 408-417.

6. C. Bertram-Kretzberg and H. Lefmann, The Algorithmic Aspects of Uncrowded Hypergraphs, SIAM Journal on Computing 29, 1999, 201-230.

7. C. Bertram-Kretzberg, T. Hofmeister and H. Lefmann, An Algorithm for Heilbronn's Problem, SIAM Journal on Computing 30, 2000, 383-390.

8. P. Brass, An Upper Bound for the d-Dimensional Analogue of Heilbronn's Triangle Problem, SIAM Journal on Discrete Mathematics 19, 192-195, 2005.

9. B. Chazelle, Lower Bounds on The Complexity of Polytope Range Searching, Journal of the American Mathematical Society 2, 1989, 637-666.

10. R. A. Duke, H. Lefmann and V. Rödl, On Uncrowded Hypergraphs, Random Structures \& Algorithms 6, 1995, 209-212.

11. A. Fundia, Derandomizing Chebychev's Inequality to find Independent Sets in Uncrowded Hypergraphs, Random Structures \& Algorithms, 8, 1996, 131-147.

12. T. Jiang, M. Li and P. Vitany, The Average Case Area of Heilbronn-type Triangles, Random Structures \& Algorithms 20, 2002, 206-219.

13. J. Komlós, J. Pintz and E. Szemerédi, On Heilbronn's Triangle Problem, Journal of the London Mathematical Society, 24, 1981, 385-396.

14. J. Komlós, J. Pintz and E. Szemerédi, A Lower Bound for Heilbronn's Problem, Journal of the London Mathematical Society, 25, 1982, 13-24.

15. H. Lefmann, On Heilbronn's Problem in Higher Dimension, Combinatorica 23, 2003, 669-680.

16. H. Lefmann, Distributions of Points in the Unit-Square and Large k-Gons, Proceedings '16th ACM-SIAM Symposium on Discrete Algorithms SODA'2005, ACM and SIAM, 241-250.

17. H. Lefmann, Distributions of Points and Large Convex Hulls of $k$ Points, Proceedings Second Int. Conference 'Algorithmic Aspects in Information and Management AAIM'2006, LNCS 4041, Springer, 173-184.

18. H. Lefmann, Convex Hulls of Point Sets and Non-Uniform Uncrowded Hypergraphs, Proceedings Third Int. Conference 'Algorithmic Aspects in Information and Management AAIM'2007, to appear.

19. K. F. Roth, On a Problem of Heilbronn, Journal of the London Mathematical Society 26, 1951, 198-204.

20. K. F. Roth, On a Problem of Heilbronn, II, and III, Proc. of the London Mathematical Society (3), 25, 1972, 193-212, and 543-549.

21. K. F. Roth, Estimation of the Area of the Smallest Triangle Obtained by Selecting Three out of $n$ Points in a Disc of Unit Area, Proc. of Symposia in Pure Mathematics, 24, 1973, AMS, Providence, 251-262.

22. K. F. Roth, Developments in Heilbronn's Triangle Problem, Advances in Mathematics, 22, 1976, 364-385.

23. W. M. Schmidt, On a Problem of Heilbronn, Journal of the London Mathematical Society (2), 4, 1972, 545-550.

24. J. Spencer, Turán's Theorem for $k$-Graphs, Discrete Mathematics, 2, 1972, 183186. 